\title{
IMPACTOS DAS TARIFAS COMERCIAIS E DOS CUSTOS DE TRANSPORTE NO MERCADO DE ETANOL
}

\author{
Profa. Dra. Jamile de Campos Coleti \\ Universidade do Estado de Minas Gerais \\ Unidade Frutal - Av. Professor Mário Palmerio, 1001 Universitário, Frutal - MG, 38200-000 \\ jamile.coleti@uemg.br \\ Profa. Dra. Andrea Leda Ramos de Oliveira \\ Universidade Estadual de Campinas \\ Faculdade de Engenharia Agrícola - FEAGRI, Av. Cândido Rondon, 501 - Barão Geraldo \\ 13083-875 - Campinas/SP \\ Andrea.oliveira@feagri.unicamp.br
}

Profa. Dra. Ana Paula Milanez

Universidade Tecnológica Federal do Paraná

Campus Ponta Grossa - Av. Monteiro Lobato, Ponta Grossa - PR, 84016-210

apmilanez@gmail.com

Prof. Dr. Jaim José da Silva Junior

Universidade de Brasília

Campus Universitário Darcy Ribeiro, Brasília-DF | CEP 70910-900

jaimjunior@gmail.com

\section{RESUMO}

Num contexto altamente competitivo ocasionado pela globalização, o agronegócio brasileiro é considerado o setor que insere o Brasil na dinâmica econômica mundial. Com foco no etanol, este artigo busca analisar e discutir os efeitos dos custos logísticos e das tarifas comerciais que incidem sobre o etanol no mercado nacional e internacional. Através do desenvolvimento de um modelo de equilíbrio parcial formulado como um Problema de Complementaridade Mista PCM, buscamos propor novas políticas de custos de forma a tornar o etanol brasileiro mais competitivo. O Brasil é o segundo maior produtor de etanol do mundo, assim, identificar onde e como podem ser formados os custos logísticos e de comercialização é de fundamental relevância para o agronegócio brasileiro. Soma-se a isso o aumento na busca mundial por energias renováveis que eleva o número e a frequência das negociações que acabam refletindo numa elevação dos custos de transação. A partir dos cenários gerados para esse artigo, a análise de sensibilidade demonstrou que quando se reduz $20 \%$ no valor das tarifas de importação e no custo de transporte o mercado brasileiro é capaz de aumentar o seu fluxo comercializado em 5,3\% isso geraria um aumento de receita de aproximadamente U\$ 334 milhões em receitas anuais. O modelo também se apresentou mais sensível quando se compara o custo das tarifas em relação ao custo de transporte. Com isso podemos configurar um novo desenho para a cadeia do etanol onde uma menor taxação do produto atrelado à baixos custos de transporte se revertam em novas oportunidades de negócios.

Palavra-chave: Simulação; Pesquisa Operacional; Comercialização; Logística; Etanol. 


\begin{abstract}
In a highly competitive context brought about by globalization, Brazilian agribusiness is considered the sector that inserts Brazil in the world economic dynamics. Focusing on ethanol, this article seeks to analyze and discuss the effects of logistics costs and commercial tariffs on ethanol in the national and international markets. Through the development of a partial equilibrium model formulated as a PCM Mixed Complementarity Problem, we seek to propose new cost policies in order to make Brazilian ethanol more competitive. Brazil is the second largest ethanol producer in the world, so identifying where and how logistics and marketing costs can be formed is of fundamental relevance to Brazilian agribusiness. Added to this is the increase in the worldwide search for renewable energies, which increases the number and frequency of negotiations, which ultimately reflect an increase in transaction costs. From the scenarios generated for this article, the sensitivity analysis showed that when the value of import tariffs and transportation costs is reduced by $20 \%$, the Brazilian market is capable of increasing its marketed flow by $5.3 \%$, which would generate a increased revenue of approximately \$ 334 million in annual revenues. The model was also more sensitive when comparing the cost of fares to the cost of transportation. With this we can set up a new design for the ethanol chain where lower product taxation coupled with low transportation costs revert to new business opportunities.
\end{abstract}

Keywords: Simulation; Operational Research; Marketing; Logistics; Ethanol.

\title{
Como Citar:
}

COELTI, Jamile de Campos; Oliveira, Andrea Leda Ramos de; MILANEZ, Ana Paula; SILVA JUNIOR, Jaim José da. Impactos das tarifas comerciais e dos custos de transporte no mercado de etanol. In: SIMPÓSIO DE PESQUISA OPERACIONAL E LOGÍSTICA DA MARINHA, 19., 2019, Rio de Janeiro, RJ. Anais [...]. Rio de Janeiro: Centro de Análises de Sistemas Navais, 2019.

\section{INTRODUÇÃO}

Nos últimos 15 anos a agricultura obteve um desempenho excepcional: a produção de cereais, oleaginosas e leguminosas aumentou 244\%, a produção da cadeia de carnes cresceu três vezes entre 2007 e 2014, todavia, os dados do Censo Agropecuário indicam uma estabilidade relativa quando se analisa a estrutura da posse da terra e no grau de concentração da produção ao mesmo tempo em que se observa aumento nos rendimentos físicos (SILVEIRA, 2017).

Contudo, como já afirmava Wedekin (2011), a agricultura é uma atividade econômica que acaba por envolver riscos elevados, onde os quatros grandes riscos são: risco da produção risco de preço, risco de crédito e risco de contrato que, embora estejam interrelacionados, devem ser tratados como mecanismos próprios.

Segundo Milanez et al. (2010), após décadas de incerteza institucional e econômica, o mercado nacional de etanol recuperou sua força em 2003, ano do advento da tecnologia de motores flexfuel. Desde então, a demanda por etanol apresentou um crescimento acumulado de aproximadamente 310\%. Este crescimento está fortemente relacionado com o advento dos veículos flexíveis, cuja evolução das vendas tem se tornado ponto fundamental da sustentação da demanda por etanol. Em 2013, a frota de veículos flex atingiu 20,8 milhões (aumento de 16\% em relação ao ano anterior), além disso, tal frota representava cerca de 
62\% da frota total de veículos licenciados no Brasil (ANTT,2016).

O objetivo deste artigo é analisar os efeitos dos custos logísticos e dos custos das transações comerciais de etanol no mercado nacional e nas exportações, avaliando os principais impactos das variáveis de tais custos na competitividade do etanol brasileiro.

Para tanto, é proposto o desenvolvimento de um modelo de equilíbrio espacial, que propicie a orientação nas discussões relacionadas aos mecanismos de comercialização.

A hipótese é de que estão presentes na cadeia do comércio nacional e internacional custos como tarifas e custos com transportes, que reúne custos das transações, e outros custos relacionados às questões estruturais de transporte e logística. Muitos desses custos, entretanto, por serem não observáveis e de difícil mensuração, permanecem inexplorados nas discussões relacionadas à comercialização, entretanto, a sua ocorrência pode interferir em resultados de pesquisas e em avaliações de políticas.

O artigo está estruturado da seguinte forma: após a introdução na seção 2 é explorada a metodologia utilizada, levantando os principais autores que vêm trabalhando a temática desde o pioneiro Paul Samuelson em 1952, ainda na seção 2 são apresentados os dados utilizados para a implementação do modelo.

A seção 3 traz os resultados do modelo expresso em formulação de cenários: cenário base e cenários futuristas onde novas políticas de comercialização e distribuição poderão ser simuladas. Na seção 4 é discutido os efeitos da adoção de novas políticas para o setor e em seguida as conclusões e contribuições do trabalho.

\section{MATERIAIS E MÉTODOS}

\subsection{MODELOS DE EQUILÍBRIO ESPACIAL}

Segundo Alvim (2003), a escolha do modelo utilizado depende do objetivo e das questões a serem respondidaspelos estudos, e ainda, das informações disponíveis para o desenvolvimento do modelo.

Como visto em Enke (1951) se há várias regiões comercializando um produto homogêneo e cada região possui um mercado distinto, as regiões estão separadas entre si, mas não isoladas. Para cada região são conhecidas as funções de produção local, preço local e consequentemente a diferença entre o que é “exportado" e o que é “importado" para cada região.

De forma simplificada Samuelson (1952) propõe um modelo de equilíbrio através da programação linear utilizando os excedentes dos produtores e os excedentes de consumo menos os custos com transporte.

Takayama e Judge (1971) a partir da formulação de Samuelson desenvolveram um modelo que buscava solucionar as condições de equilíbrio espacial envolvendo commodities transacionadas entre diferentes regiões. De acordo com Oliveira e Alvim (2017), os modelos de equilíbrio parcial permitem resolver problemas de comércio entre diferentes regiões (separadas espacialmente) que apresentam diferentes ofertas, demandas e fluxos comerciais.

Os Alguns exemplos da utilização do modelo de equilíbrio espacial para analisar a competição inter-regional de produtos agrícolas podem ser encontrados em Von Oppen e Scott (1976), Waquil e Cox (1995), Yang et al. (2002), Souza (2008), Malý et al. (2011), Holderieath et al. (2018).

Para a formulação de modelos na área agrícola, várias metodologias têm sido utilizadas, entre elas pode-se destacar a utilização dos modelos de equilíbrio parcial e espacial, os quais têm origem em Samuelson (1952) e Enke (1951), que demonstraram como este tipo de problema pode ser resolvido através do uso de programação matemática. Takayama e Judge (1971) usaram um preço linear dependente e funções de demanda e oferta 
estendidas da formulação de Samuelson, para obter dimensões espaciais e intertemporais de preço, produção, fator de uso e consumo determinados através de um quadro de programação quadrática. Eles desenvolveram um algoritmo capaz de solucionar as condições de equilíbrio espacial envolvendo diversas commodities transacionadas entre muitas regiões.

Recentemente, Paris et al. (2011) desenvolvem um modelo de calibração para modelos de equilíbrio espacial. Segundo os autores, as especificações de programação matemática exibem certas divergências entre os resultados estimados e os dados observados de oferta, de demanda e do fluxo de comércio entre os países. Essas diferenças podem ser impostas à imprecisão dos custos que ocorrem nas transações comerciais ou na imprecisão das medidas dos parâmetros das funções de oferta e demanda, ou então, tal imprecisão pode ser por ambos os motivos. A utilização do modelo sem a correção de tais diferenças pode resultar em geração de políticas distorcidas.

Definidas as especificações matemáticas e com a ajuda de softwares computacionais é possível estimar o ponto para onde as variáveis utilizadas no problema convergem e que se refere ao ótimo da questão.

\subsection{Problema de Complementariedade Mista (PCM)}

O modelo de Complementaridade Mista tem a vantagem de permitir a incorporação de tarifas, quotas-tarifárias com mais facilidade ao modelo. A solução sugerida através deste modelo é capaz de determinar os fluxos comerciais entre as regiões, detalhando as melhores opções para produção, processamento, armazenamento e distribuição, levando em consideração as mudanças na logística de transporte.

De acordo com Waquil (2000) em uma situação hipotética onde não há comércio entre duas regiões, os preços e quantidades em equilíbrio são determinados pela intersecção das curvas de oferta e demanda locais. Nesse sentido, as preferências dos consumidores serão diferentes entre as regiões, resultando em preços de equilíbrio diferentes para cada uma das regiões. Se trabalharmos com uma segunda proposição, com livre comércio e na ausência de custo de transportes, haverá comércio até que os preços das regiões sejam iguais.

O PCM pode ser descrito conforme as equações, adaptadas de Paris et al. (2011) e Caetani (2014), que representa as condições que permitem obter os preços, as quantidades produzidas e consumidas e os fluxos comerciais entre as regiões, levando em consideração os custos logísticos e de transações dos mercados. Deste modo O PCM para a comercialização do etanol é apresentado a seguir, considerando regiões de demanda, interna e internacional.

Índices:

$j$ : regiões de oferta de etanol $J=\{1,2, \ldots, 6\}$

$i$ : regiões de demanda interna para o etanol $\mathrm{I}=\{1,2,3\}$

$k$ : regiões de demanda internacional para o etanol $\mathrm{K}=\{1,2,3,4\}$

Onde:

$\mathrm{y}_{\mathrm{i}}$ é a quantidade demandada na região $i$

$\mathrm{Z}_{\mathrm{j}}$ é a quantidade ofertada na região $\mathrm{j}$

$g_{k}$ é a quantidade demandada na região $k$

$x_{j i}\left(x_{j k}\right)$ é a quantidade comercializada entre as regiões $j$ e $i(k)$;

$t_{j i}\left(t_{j k} \dot{i}\right.$ é o custo de trasnporte entre as regiões $j$ e $i(k)$;

$\operatorname{trt}_{j i}\left(\operatorname{trt}_{j k}\right)$ é a tarifa cobrada sobre o produto que se originou na região $j$ e tem como destino a região $i(k)$;

$\varphi_{j}$ é o multiplicador de Lagrange (preço-sombra da região de oferta $j$ ); 
$\lambda_{i}$ é o multiplicador de Lagrange (preço-sombra da região de demanda i);

$\delta_{k}$ é o multiplicador de Lagrange (preço-sombra da região de demanda internacional k);

$\gamma_{j i}\left(\gamma_{j k}\right)$ é o multiplicador de Lagrange (preço-sombra na região de oferta $\mathrm{j}$ e de demanda $i$ $(k)$.

$$
\begin{aligned}
& y_{i} \leq \sum_{j=1}^{6} x_{j i} \lambda_{i} \geq 0\left[\sum_{j=1}^{6} x_{j i}-y_{i}\right] \lambda_{i}=0 \forall i \in I(1) \\
& g_{k} \leq \sum_{j=1}^{6} x_{j k} \delta_{k} \geq 0\left[\sum_{j=1}^{6} x_{k j}-g_{k}\right] \delta_{k}=0 \forall k \in K(2) \\
& \sum_{i=1}^{3} x_{j i}+\sum_{k=1}^{4} x_{j k} \leq z_{j} \varphi_{j} \geq 0\left[z_{j}-\left(\sum_{i=1}^{3} x_{j i}+\sum_{k=1}^{4} x_{j k}\right)\right] \varphi_{j}=0 \forall j \in j(3) \\
& \lambda_{i} \leq \varphi_{j}+t_{j i} x_{j i} \geq 0\left(\left(t_{j i}+\varphi_{j}\right)\left(1+\operatorname{trt}_{j i}\right)-\lambda_{i}\right) x_{j i}=0 \forall i \in I, j \in J(4) \\
& \delta_{k} \leq \varphi_{j}+t_{j k} x_{j k} \geq 0\left(\left(t_{j k}+\varphi_{j}\right)\left(1+\operatorname{trt}_{j k}\right)-\delta_{k}\right) x_{j k}=0 \forall k \in k, j \in J(5) \\
& y_{i}, z_{j}, g_{k}, x_{j i}, x_{j k} \geq 0 \forall i \in I, j \in J, k \in K(6)
\end{aligned}
$$

A equação (6) garante a não-negatividade das quantidades produzidas, consumidas e comercializadas.

A solução ótima desse PCM é obtida a partir da convergência das equações (1), (2) e (3), na equação (4) há uma condição de complementariedade onde o preço de mercado da região de demanda i deve sempre ser menor do que o preço de oferta da região j somado os custos de transporte e de transação e da tarifa até a região de demanda. A equação (5) representa a mesma complementariedade só que para a demanda internacional.

Caso contrário, se não houver fluxo comercial, isto significa que o preço na região de demanda é menor que o preço na região de oferta somado aos custos de transporte e transação e da tarifa.

Originalmente, Paris et al. (2011) utilizam o primal-dual que é calculado com base nas condições de Karush-Kuhn-Tucker. Os resultados obtidos através do modelo utilizado pelos autores são exatamente iguais ao PCM, possibilitando tal adaptação. As estimativas são obtidas através de duas fases de PCM: inicialmente serão estimados os custos das transações, as quantidades e os preços de oferta e de demanda, as quantidades do fluxo comercial e os custos logísticos, onde os custos das transações são endógenos e as outras variáveis são exógenas ao modelo. A variável que representa os custos das transações comerciais entre a região produtora j e consumidora i (k) é uma variável endógena ao modelo.

Após a convergência das equações (1), (2), e (3) do PCM é incluída uma quarta equação cujo objetivo é estimar os custos de transação como proposto por Paris et al. (2011). Baseado nos estudos de Samuelson (1952) e Takayama e Judge (1994) os autores desenvolveram um modelo de calibração de comércio onde introduziu nas equações de programação matemática uma variável de ajuste para os problemas de otimização.

De acordo com Paris et al. (2011) há uma certa diferença dentre os problemas matemáticos com relação aos resultados estimados e os observados de oferta e demanda e do fluxo de comércio entre diferentes regiões, isso pode ocorrer devido à difícil mensuração dos 
custos incorridos nas transações o que compromete os resultados.

Na primeira fase são estimados os custos de transação, as quantidades do fluxo comercial, os preços de oferta e de demanda e os custos de transporte. Os custos de transação representam uma variável endógena $\left(\gamma_{-} \mathrm{ji}, \gamma_{-} \mathrm{jk}\right)$ e as demais variáveis são exógenas.

Para estimar o fluxo comercial entre as regiões de excesso de oferta e excesso de demanda na primeira fase foi desenvolvida uma matriz origem-destino. As matrizes origemdestino para estimativa dos fluxos de exportação utilizado nesta tese é uma técnica usualmente utilizada na Engenharia de Transportes e que tem ampla aplicabilidade em estudos econômicos (HAMERSLAG e IMMERS, 1998). Existem diversos estudos que utilizaram uma matriz O-D com intuito de minimizar o custo de transferência de diversos tipos de cargas para diversos locais, com destaque: Oliveira (2007); Figueiredo et al. (2005); Martins e Caixeta Filho (1998) e Ortúzar e Willumsem (1995).

O conjunto de equações que compõe a primeira fase para a obtenção da variável custos de transação $\gamma_{-}$ji e $y_{[} j \mathrm{jk}$ é:

$$
\begin{aligned}
& y_{i} \leq \sum_{j=1}^{6} x_{j i} \lambda_{i} \geq 0\left[\sum_{j=1}^{6} x_{j i}-y_{i}\right] \lambda_{i}=0 \forall i \in I(7) \\
& g_{k} \leq \sum_{j=1}^{6} x_{j k} \delta_{k} \geq 0\left[\sum_{j=1}^{6} x_{j k}-g_{k}\right] \delta_{k}=0 \forall k \in K(8) \\
& \sum_{k=1}^{4} x_{j k}+\sum_{i=1}^{3} x_{j i} \leq z_{j} \varphi_{j} \geq 0\left[z_{j}-\left(\sum_{k=1}^{4} x_{j k}+\sum_{i=1}^{3} x_{j i}\right)\right] \varphi_{j}=0 \forall j \in J(9) \\
& \lambda_{i} \leq \varphi_{j}+t_{j i}+\gamma_{j i} x_{j i} \geq 0\left(\gamma_{j i}+\left(t_{j i}+\varphi_{j}\right)\left(1+\operatorname{trt}_{j i}\right)-\lambda_{i}\right) x_{j i}=0 \forall i \in I, j \in J(10) \\
& \delta_{k} \leq \varphi_{j}+t_{j k}+\gamma_{j k} x_{j k} \geq 0\left(\gamma_{j k}+\left(t_{j k}+\varphi_{j}\right)\left(1+t r t_{j k}\right)-\delta_{k}\right) x_{j k}=0 \forall k \in K, j \in J(11)
\end{aligned}
$$

A condição de complementaridade dada pela equação (10) assume que o preço de mercado da região de demanda j deve ser menor do que o preço de oferta da região i somado os custos de transporte, aos custos das transações, e da tarifa que possui uma alíquota para cada região.

Na segunda fase, são introduzidos na programação os custos de transação gerados na primeira fase e são estimados exogenamente os custos de transação com as quantidades e preços de oferta e demanda - que passam a ser uma variável exógena. Nessa fase a variável que representa os custos de transações, gerados na primeira é representado por $\hat{\gamma} \mathrm{ji}$ e $\hat{\gamma} \mathrm{j}$ k e é adicionada no modelo, sendo estimada exogenamente com as quantidades e preços de oferta e demanda.

A expressão matemática do PCM na segunda fase é dada por:

$$
\begin{aligned}
& y_{i} \leq \sum_{j=1}^{6} x_{j i} \lambda_{i} \geq 0\left[\sum_{j=1}^{6} x_{j i}-y_{i}\right] \lambda_{i}=0 \forall i \in I(12) \\
& g_{k} \leq \sum_{j=1}^{6} x_{j k} \delta_{k} \geq 0\left[\sum_{j=1}^{6} x_{j k}-g_{k}\right] \delta_{k}=0 \forall j \in J(13) \\
& \sum_{k=1}^{4} x_{j k}+\sum_{i=1}^{3} x_{j i} \leq z_{j} \varphi_{j} \geq 0\left[z_{j}-\left(\sum_{k=1}^{4} x_{j k}+\sum_{i=1}^{3} x_{j i}\right)\right] \varphi_{j}=0 \forall j \in J(14) \\
& \lambda_{i} \leq \varphi_{j}+t_{j i}+\gamma_{j i} x_{j i} \geq 0\left(\hat{\gamma}_{j i}+\left(t_{j i}+\varphi_{j}\right)\left(1+t r t_{j i}\right)-\lambda_{i}\right) x_{j i}=0 \forall i \in I, j \in J(15) \\
& \delta_{k} \leq \varphi_{j}+t_{j k}+\gamma_{j k} x_{j k} \geq 0\left(\hat{\gamma}_{j k}+\left(t_{j k}+\varphi_{j}\right)\left(1+t r t_{j k}\right)-\delta_{k}\right) x_{j k}=0 \forall k \in K, j \in J(16)
\end{aligned}
$$

A solução é obtida a partir da convergência das equações complementares (12), (13) 
e (14). Para cumprir com a condição de complementaridade apresentada na equação (15) o preço de mercado da região de demanda i deve ser menor que o preço de oferta da região j somado os custos de transporte, os custos das transações, e a tarifa cobrada por produto. A equação (16) representa a mesma complementariedade só que para a demanda internacional

Com base com resultados obtidos nessa segunda fase, é possível propor cenários base e alternativos, levando em conta a manutenção ou eliminação de tarifas e custos das transações.

\subsection{DESCRIÇÃO DOS DADOS}

As regiões foram divididas em Macro Regiões para facilitar o entendimento, o Estado de São Paulo foi dividido em três Macro Regiões: Macro 1, Macro 2 e Macro 3, os Estados de Sergipe, Rio Grande do Norte e Bahia agrupadas formam a região Nordeste e os Estados de Paraná, Rio Grande do Sul e Santa Catarina formam a Região Sul. O Quadro 1 mostra como foram feitos os agrupamentos.

Quadro 1. Composição das Macro-Regiões estudadas

\begin{tabular}{|c|c|}
\hline Macrorregião & Microrregiões/Estados envolvidos \\
\hline Macro 1 & $\begin{array}{c}\text { São José do Rio Preto } \\
\text { Ribeirão Preto }\end{array}$ \\
\hline Macro 2 & $\begin{array}{c}\text { Araraquara } \\
\text { Piracicaba } \\
\text { Campinas }\end{array}$ \\
\hline Macro 3 & $\begin{array}{c}\text { Presidente Prudente } \\
\text { Marilia } \\
\text { Assis } \\
\text { Itapetininga } \\
\text { Araçatuba } \\
\text { Bauru }\end{array}$ \\
\hline Mato Grosso do Sul & Mato Grosso do Sul \\
\hline Minas Gerais & Minas Gerais \\
\hline Goiás & Goiás \\
\hline Nordeste & $\begin{array}{c}\text { Sergipe } \\
\text { Bahia } \\
\text { Rio Grande do Norte } \\
\end{array}$ \\
\hline Sul & $\begin{array}{c}\text { Paraná } \\
\text { Santa Catarina } \\
\text { Rio Grande do Sul }\end{array}$ \\
\hline Rio de Janeiro & Rio de Janeiro \\
\hline
\end{tabular}

A Macrorregião 1 representa as mesorregiões ${ }^{1}$ de São José do Rio Preto e Ribeirão Preto. Essa região representa cerca de $24 \%$ de todo etanol produzido no Brasil. As mesorregiões que compõem a Macrorregião 2 são: Araraquara, Piracicaba e Campinas, que juntas produzem aproximadamente $13 \%$ do total de etanol. A Macrorregião 3 é composta

1 Mesorregião é uma divisão regional do Instituto Brasileiro de Geografia e Estatística (IBGE), a divisão foi feita de acordo com as seguintes dimensões: o processo social como determinante, o quadro natural como condicionante e a rede de comunicação e de lugares como elemento da articulação espacial (IBGE, 2014). 
pelas seguintes mesorregiões: Araçatuba, Bauru, Presidente Prudente, Marília, Assis e Itapetininga, que juntas são responsáveis por cerca de $14 \%$ da produção total de etanol no Brasil. Os estados de Goiás e Mato Grosso do Sul juntos, produzem cerca de 35\% do total brasileiro de etanol (UNICA, 2018).

As regiões Nordeste, Sul e o estado do Rio de Janeiro, são responsáveis por absorver grande parte do excedente do etanol produzido na região Centro-Sul, pois não produzem a quantidade suficiente de etanol para seu auto abastecimento e, portanto, são considerados como regiões de destino.

Os dados de produção e consumo compõe o critério adotado para determinação de quais são as regiões de excesso de oferta e regiões de demanda, de forma que se subtraímos a quantidade consumida da quantidade produzida e ela possuir um excedente representativo, essa região será de excesso de oferta, por outro lado, se subtrair o consumo da produção e seu volume de produção não for o suficiente para abastecer a região, essa será uma região de excesso de demanda.

Os dados de produção e consumo tiveram como base o ano de 2016, os dados de produção foram extraídos da UNICA (UNICA, 2018), enquanto os dados de consumos foram extraídos da Agência Nacional de Petróleo (ANP, 2018). A Tabela 1 apresenta os dados de produção e consumo e a classificação da região como excesso de oferta e excesso de demanda.

Tabela 1. Dados de produção e consumo para etanol em $2016\left(\mathrm{mil} \mathrm{m}^{3}\right)$

\begin{tabular}{c|c|c|c}
\hline Estado/Safra & Produção & Consumo & Classificação \\
\hline São Paulo & 14.577 & 11.054 & Oferta \\
Goiás & 4.689 & 1.472 & Oferta \\
Mato Grosso do Sul & 2.777 & 316 & Oferta \\
Minas Gerais & 3.069 & 2.666 & Oferta \\
Paraná & 1.574 & 2.023 & Consumo \\
Bahia & 221 & 903 & Consumo \\
Rio de Janeiro & 59 & 1.206 & Consumo \\
Sergipe & 112 & 133 & Consumo \\
Rio Grande do Norte & 89 & 231 & Consumo \\
Rio Grande do Sul & 4 & 1.004 & Consumo \\
Santa Catarina & 0 & 804 & Consumo \\
\hline
\end{tabular}

Fonte: ÚNICA (2018) e ANP (2018)

Em relação à demanda internacional, foram selecionados os países: Estados Unidos, Coreia do Sul e Holanda além do Resto do Mundo². Os Estados Unidos é o maior produtor de etanol do mundo e tem a maior participação nas importações mercado. De acordo com dados de exportação, a Coreia do Sul e Holanda ocupam a posição de segundo e terceiro maiores importadores de etanol brasileiro, respectivamente (BRASIL, 2016).

Segundo Krugman e Wells (2012) a elasticidade-preço de oferta é a medida da sensibilidade da quantidade ofertada de um bem em relação ao seu preço e a elasticidadepreço da demanda é a razão entre a mudança percentual na quantidade demandada e a mudança percentual no preço, à medida que há alterações na curva de demanda. As informações de elasticidade-preço da oferta e da demanda para o etanol foram obtidas nos estudos de Beiral (2011), Boff (2009), e em Luchansky e Monks (2009) e sã apresentados na Tabela 2.

2 O Resto do Mundo representa o conjunto dos demais países que recebem etanol brasileiro. 
Os preços do etanol no mercado doméstico foram obtidos na ANP (2018) e para o mercado internacional na Renewable Fuels Association (RFA, 2018) e expressam o valor do $\mathrm{m}^{3}$ do etanol em US\$.

Tabela 2. Dados de entrada do modelo

\begin{tabular}{c|c|c}
\hline Origem/Destino & Preço & Elasticidades-preço \\
\hline Macro 1 - O & 441,23 & 0,21 \\
Macro 2 - O & 475,97 & 0,21 \\
Macro 3 - O & 467,95 & 0,21 \\
Goiás - O & 470,80 & 0,21 \\
Mato Grosso do Sul - O & 556,70 & 0,21 \\
Minas Gerais - O & 506,19 & 0,21 \\
Nordeste - D & 498,20 & 0,74 \\
Sul - D & 496,40 & 0,58 \\
Rio de Janeiro - D & 488,30 & 0,58 \\
Estados Unidos - D & 375,13 & 2,92 \\
Holanda - D & 375,13 & 2,92 \\
Coréia do Sul - D & 375,13 & 2,92 \\
Resto do Mundo - D & 400,00 & 2,92 \\
\hline
\end{tabular}

Fonte: Elaborado a partir de Beiral (2011); Boff (2009);Luchansky e Monks (2009), ANP (2017) e RFA (2018).

As tarifas para o mercado doméstico foram calculadas levando em consideração a tributação incidente sobre o etanol em um levantamento feito com especialistas do setor:

a) Imposto sobre Circulação de Mercadorias e Serviços (ICMS) alíquota praticada em 2016 sobre o valor da mercadoria entre os estados. Todas as regiões que movimentam para a Região Sul e para o Estado do Rio de Janeiro apresentam alíquota de 12\%; Já para o Nordeste, os fluxos com origem no estado de São Paulo e Minas Gerais apresentam alíquota de 7\% enquanto os fluxos com origem nos estados de Goiás e Mato Grosso do Sul possuem alíquota de $12 \%$.

b) Programa de Integração Social (PIS): valor fixo de $\mathrm{R} \$ 21,43$ por $\mathrm{m}^{3}$ (CEPEA,

c) Contribuição para Financiamento da Seguridade Social (COFINS): valor fixo de $\mathrm{R} \$ 98,57$ por $\mathrm{m}^{3}$ (CEPEA, 2018).

Para o mercado internacional foram consideradas as tarifas de importação incidentes para o etanol em 2016, os dados da Holanda mostram uma taxação de $27 \%$ sobre o etanol brasileiro (NETHERLANDS, 2018), de 2,5\% nos Estados Unidos (USDA, 2017) e de 15\% para a Coréia do Sul (ITA, 2016), para o Resto do Mundo foi adotado uma tarifa média de 17\%. Os dados referentes às tarifas no mercado de etanol para ao no de 2016 são apresentados na tabela abaixo:

Tabela3. Tarifas nas regiões de estudo em $2016\left(\mathrm{US} \$ / \mathrm{m}^{3}\right)$.

\begin{tabular}{|c|c|c|c|c|c|c|c|}
\hline Origem/Destino & $\begin{array}{l}\text { Estados } \\
\text { Unidos }\end{array}$ & Holanda & $\begin{array}{l}\text { Coréia } \\
\text { do Sul }\end{array}$ & $\begin{array}{c}\text { Resto do } \\
\text { Mundo }\end{array}$ & Sul & Nordeste & $\begin{array}{l}\text { Rio de } \\
\text { Janeiro }\end{array}$ \\
\hline Macro 1 & 11,03 & 119,13 & 66,18 & 75,01 & 89,20 & 67,14 & 89,20 \\
\hline Macro 2 & 11,90 & 128,51 & 71,40 & 80,91 & 93,37 & 69,57 & 93,37 \\
\hline Macro 3 & 11,70 & 126,35 & 70,19 & 79,55 & 92,41 & 69,01 & 92,41 \\
\hline
\end{tabular}




\begin{tabular}{c|cccc|ccc} 
Goiás & 11,77 & 127,12 & 70,62 & 80,04 & 92,75 & 92,75 & 92,75 \\
Mato Grosso do & & & & & & & \\
Sul & 13,92 & 150,31 & 83,51 & 94,64 & 103,06 & 103,06 & 103,06 \\
Minas Gerais & 12,65 & 136,67 & 75,93 & 86,05 & 97,00 & 71,69 & 97,00 \\
\hline
\end{tabular}

Fonte: Dados da pesquisa (2018).

Os custos de transporte para o mercado doméstico foram obtidos no informe SIFRECA (2017) e os do mercado internacional ${ }^{3}$ foram calculados a partir de OCDE (2017) e são apresentados na Tabela 4.

Tabela 4. Custos de transporte nas regiões de estudo (em US\$/ $\mathrm{m}^{3}$ )

\begin{tabular}{c|cccc|ccc}
\hline Origem/Destino & $\begin{array}{c}\text { Estados } \\
\text { Unidos }\end{array}$ & Holanda & $\begin{array}{c}\text { Coréia } \\
\text { do Sul }\end{array}$ & $\begin{array}{c}\text { Resto } \\
\text { do } \\
\text { Mundo }\end{array}$ & Sul & Nordeste & $\begin{array}{c}\text { Rio de } \\
\text { Janeiro }\end{array}$ \\
\hline Macro 1 & 49,85 & 55,14 & 55,15 & 55,15 & 40,60 & 76,70 & 33,00 \\
Macro 2 & 59,81 & 49,84 & 49,85 & 49,85 & 35,50 & 83,20 & 28,10 \\
Macro 3 & 66,40 & 59,80 & 59,81 & 59,81 & 39,70 & 84,20 & 40,80 \\
Goiás & 66,40 & 66,39 & 66,40 & 66,40 & 52,80 & 76,00 & 45,50 \\
Mato Grosso do & & & & & & & \\
Sul & 74,76 & 74,75 & 74,76 & 74,76 & 42,40 & 101,10 & 55,90 \\
Minas Gerais & 61,75 & 61,74 & 61,75 & 61,75 & 49,50 & 70,40 & 41,90 \\
\hline
\end{tabular}

Fonte: SIFRECA (2017), OCDE (2017).

\section{RESULTADOS E DISCUSSÃO}

O método de calibração utilizado neste estudo foi desenvolvido por Anania (2011) que utiliza o método de ajustes através da variável de custos das transações que é gerada na primeira fase do problema e introduzida como uma variável exógena na segunda fase das estimativas. A partir deste procedimento são propostos os cenários bases e alternativos que serão apresentados a seguir.

\subsection{CENÁRIO baSE}

O cenário base foi gerado na segunda fase do modelo a partir das estimativas obtidas na primeira fase e da calibração do modelo onde os resultados servem como parâmetros para propor cenários alternativos.

As estimativas do cenário base destacam a solução ótima do problema de forma a estimar os fluxos de etanol comercializados entre diferentes regiões para o mercado interno e para o mercado externo considerando a base de dados do ano de 2016.

Os custos das transações estimados na primeira fase são introduzidos no modelo, participando como uma variável exógena e servindo de elemento para a calibração do modelo. A tabela 5 mostra as quantidades comercializadas entre as regiões para o mercado interno e externo.

Podemos observar que assim como é praticado atualmente a maior parte do fluxo (85\%) foi absorvido pelo mercado interno, que o Nordeste é abastecido pelo estado de São Paulo e Minas Gerais, que a região Sul é abastecida pelos estados de Goiás e Mato Grosso do Sul e que o Rio de Janeiro é abastecido pela Macro-1 e Goiás.

Tabela 5 - Cenário base no mercado interno e externo $(\mathrm{em} \mathrm{mil} \mathrm{m³})$ 


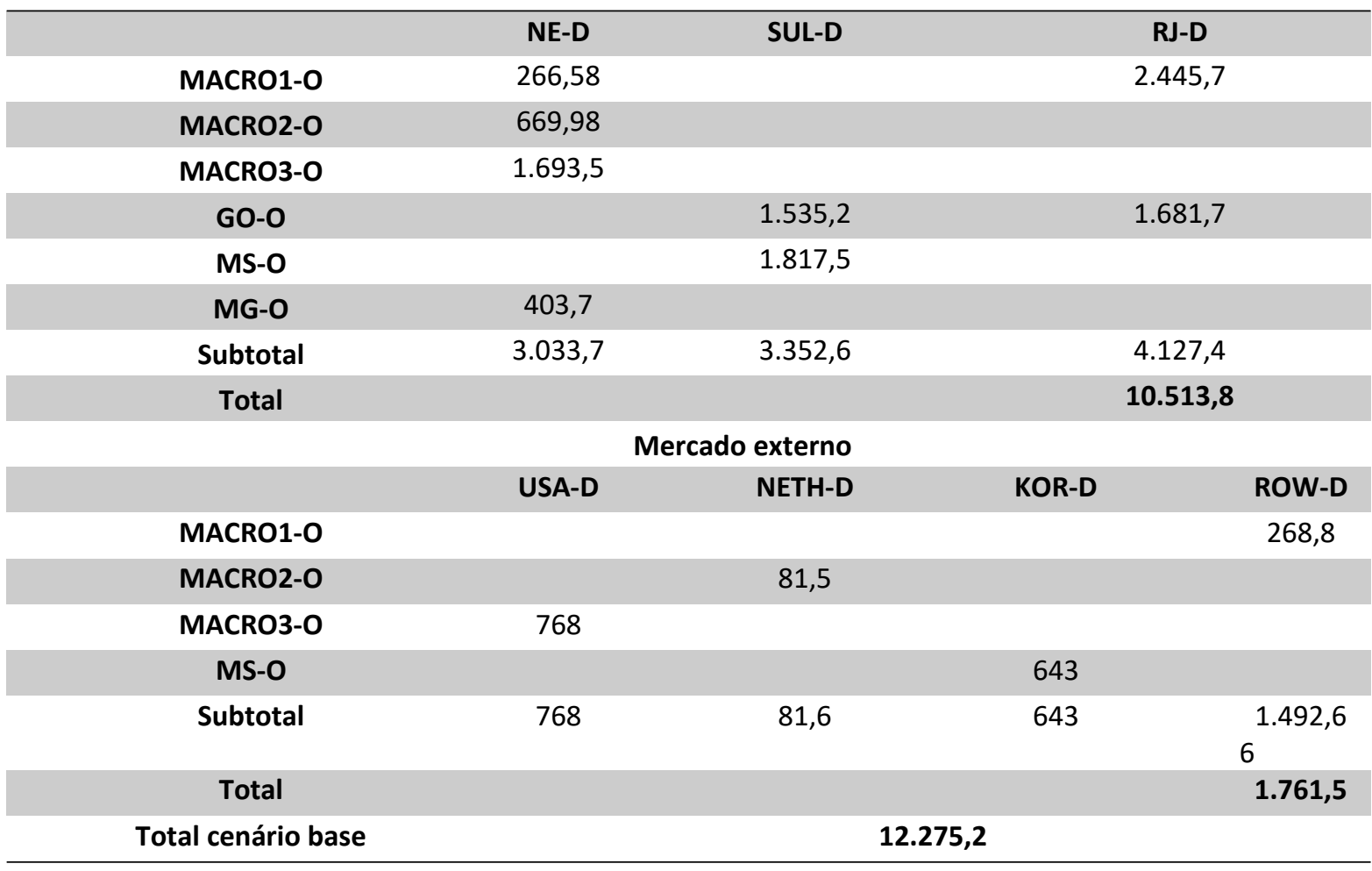

Fonte: Dados da pesquisa (2019).

As estimativas do mercado internacional indicam que as regiões de oferta Goiás e Minas Gerais não apresentam rota competitiva com destino internacional, podemos atribuir esse fenômeno ao fato de que $90 \%$ do etanol é transportado via Porto de Santos e essas duas regiões são mais distantes. Além disso, o fluxo com destino ao mercado externo representa apenas $15 \%$ do total de etanol comercializado no Brasil. Ao somarmos o mercado interno com exportação temos um total de 12,27 milhões de $\mathrm{m}^{3}$ comercializados.

\subsection{CEnÁRios ALternativos}

Os cenários alternativos são simulações de variáveis que possam interferir no mercado de etanol. Os resultados de cada cenário vão poder guiar estratégias futuras para tomada de decisão por parte de diversos players do setor.

São propostos três cenários a priori, embora para a realização desse artigo foram testados cerca de 32 cenários, os cenários que foram descartados não apresentavam real chance de se tornarem políticas comerciais pois ou tinham um pequeno efeito sobre o fluxo comercializado ou por questões econômicas se tratavam de cenários irreais.

O primeiro cenário descartado testava a possibilidade de diminuir $10 \%$ no custo de transporte, $10 \%$ no custo das tarifas e $10 \%$ no custo de transporte e de tarifa. Esse cenário foi descartado pois a variação no fluxo comercializado foi quase que insignificante.

O segundo cenário descartado testava a possibilidade de diminuir $40 \%$ no custo de transporte, $40 \%$ no custo das tarifas e $40 \%$ no custo de transporte e de tarifa. Esse cenário foi descartado pois diminuir o custo de transporte em $40 \%$ não seria negociável com as empresas transportadoras, já que os demais custos que uma empresa transportadora tem se manteriam inalterados. Além disso, uma diminuição de $40 \%$ da tarifa inclui uma redução de $40 \%$ na arrecadação de pis/cofins e ICMS, embora haja uma discussão por parte do setor para uniformizar a tarifa de ICMS, possivelmente essa tarifa não seria fixada nos $40 \%$ a menos do que se é praticado hoje. 
Os cenários propostos então são os seguintes:

Cenário 1: Redução de $20 \%$ no custo de transporte; Aumento de $20 \%$ no Custo de Transporte;

Cenário 2: Redução de 20\% no valor da tarifa; Aumento de 20\% na tarifa;

Cenário 3: Redução de $20 \%$ no valor da tarifa e no custo de transporte; Aumento de $20 \%$ na tarifa e no custo de transporte;

O valor de $20 \%$ foi definido em função da variação do custo de transporte que do ano de 2016 variou 20 pontos percentuais para baixo e 20 pontos percentuais para cima quando se compara a mesma rota no ano de 2018, podemos observar esse efeito na Tabela 6.

Tabela 6 . Variação do valor de frete entre 2016-2018

\begin{tabular}{cc|rr|c}
\hline Origem & Destino & \multicolumn{1}{c|}{2016} & \multicolumn{1}{c|}{2018} & Variação \\
\hline Macro 1/Ribeirão Preto & Santos & 53,13 & 68,69 & $23 \%$ \\
Macro 2/Piracicaba & Santos & 45,69 & 61,00 & $25 \%$ \\
Macro 3/Araçatuba & Santos & 103,15 & 145,16 & $29 \%$ \\
Macro 1/Ribeirão Preto & Santos & 106,83 & 90,45 & $-15 \%$ \\
GO/Itumbiara & NE/Salvador & 354,00 & 265,00 & $-25 \%$ \\
Macro 1/Ribeirão Preto & Sul/Ponta Grossa & 109,50 & 120,00 & $9 \%$ \\
MG/Uberaba & Santos & 134,56 & 185,00 & $27 \%$ \\
MS/Dourados & Sul/Ponta Grossa & 90,23 & 120,00 & $25 \%$ \\
Macro 3/Araçatuba & RJ/Duque de Caxias & 140,00 & 137,50 & $-2 \%$ \\
Variação média & & & & $\mathbf{2 0 \%}$ \\
\hline
\end{tabular}

Fonte: SIFRECA (2018).

A tabela 7 apresenta os volumes movimentados para cada cenário alternativo e mostra a variação deles em relação ao cenário base. O Cenário 1 mostrou que quando se aumenta em $20 \%$ o custo de transporte, o fluxo comercializado diminui $3,7 \%$ e que quando se reduz $20 \%$ o custo de transporte, o fluxo de etanol comercializado aumenta em $0,9 \%$.

No cenário 2 é testada variações nas tarifas, quando se aumenta $20 \%$ nas tarifas o fluxo comercializado se reduz em $3,9 \%$; e quando se reduz $20 \%$ no valor das tarifas há um aumento de $4,8 \%$ no fluxo comercializado. Isso nos mostra que o modelo é mais sensível às variações nas tarifas do que no custo de transporte, ou ainda, que variações nas tarifas causam um maior efeito no mercado de etanol do que o custo de transporte.

O cenário 3 é o cenário principal, pois nele testamos as variações para o custo de transporte e valor das tarifas em conjunto. O resultado é de que quando aumentamos $20 \%$ os custos de comercialização (custo de transporte + valor das tarifas), há uma redução no fluxo movimentado de $4,3 \%$ e isso representaria uma perda de US\$ 257,7 milhões nas receitas brasileiras se comparado ao cenário base. Por outro lado, uma redução de $20 \%$ nos custos de comercialização, teríamos um fluxo 5,3\% maior e, portanto, um aumento da ordem de U\$S 334 milhões.

Tabela 72. Resultado resumido dos cenários alternativos $\left(\mathrm{mil} \mathrm{m}^{3}\right)$

\begin{tabular}{c|cc|cc|cc}
\hline \multirow{2}{*}{$\begin{array}{c}\text { Cenário } \\
\text { Base }\end{array}$} & \multicolumn{2}{|c|}{ Cenário 1 } & \multicolumn{2}{c|}{ Cenário 2 } & \multicolumn{2}{c}{ Cenário 3 } \\
\cline { 2 - 7 } & CT+20 & CT-20 & TAR+20 & TAR-20 & CTTAR +20 & CTTAR -20 \\
\hline $12.275,21$ & $11.823,7$ & $12.389,0$ & $11.795,8$ & $12.899,2$ & $11.744,7$ & $12.962,8$ \\
Variação & $-3,7 \%$ & $0,9 \%$ & $-3,9 \%$ & $4,8 \%$ & $-4,3 \%$ & $5,3 \%$ \\
\hline
\end{tabular}

Fonte: Dados da pesquisa (2019). 
Para complementar o estudo uma análise de sensibilidade foi feita buscando ilustrar as variações de um cenário para outro. Como dito anteriormente, o Cenário 1 se mostrou menos significativo quando se compara com os demais, observe na Figura 1:

Figura 1. Análise de sensibilidade dos cenários alternativos

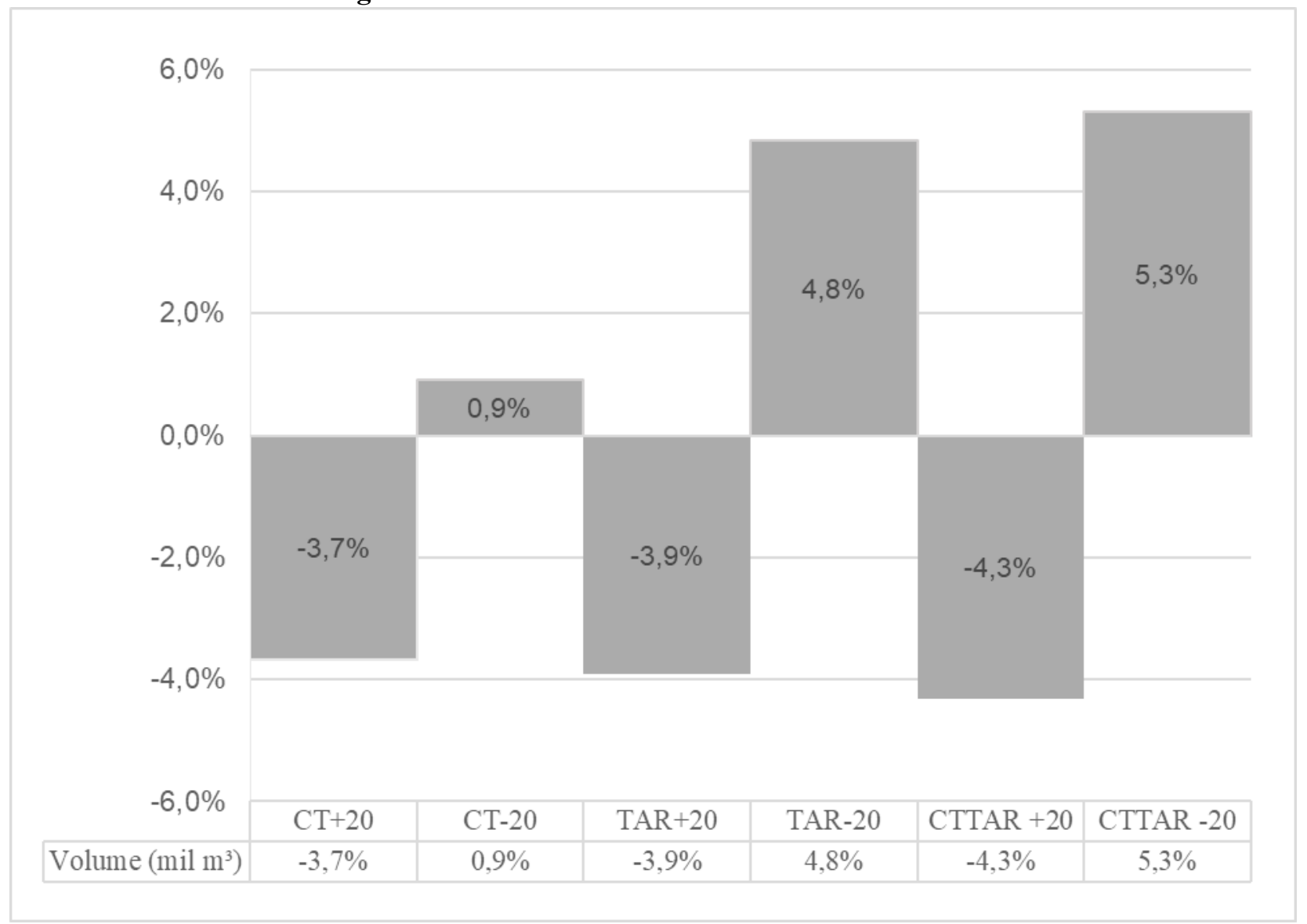

Fonte: Dados da pesquisa (2019).

\section{CONCLUSÃO}

Modelos de otimização são pouco usuais para aplicações em commodities agrícolas, recentes trabalhos têm contribuído para o setor de transportes, como é o caso da aplicação de modelos para combustíveis.

Este estudo demonstra os impactos dos custos de transporte e das tarifas no mercado interno e nas exportações do etanol brasileiro. Os resultados permitem captar esses efeitos nas principais regiões produtoras e consumidoras e nos principais países importadores e resto do mundo, de forma a medir quanto políticas de comércio e de distribuição influenciam nos fluxos comercializados.

O cenário base é um retrato da situação atual sem descontos ou acréscimos nos custos de transporte e mostra que atualmente é movimentado um total de 12,2 milhões de $\mathrm{m}^{3}$ de etanol no setor, incluindo demanda interna e comércio internacional.

Os cenários alternativos buscavam ver o efeito de novas políticas de comércio e de distribuição no segmento. No cenário 1 quando há um acréscimo de $20 \%$ no custo de transporte, ocorre uma diminuição no fluxo de transporte da ordem de $3,7 \%$, isso mostra que o mercado de etanol se retrai caso os custos de transporte aumentem. Ainda no cenário 1 , quando diminuímos o custo de transporte em $20 \%$ o fluxo aumenta apenas $0,9 \%$, não chega 
nem a 1 ponto percentual. Uma análise geral desse cenário é que ele é mais sensível à aumentos no custo de transporte.

Em relação ao cenário 2 a proposta foi analisar alteração nas tarifas de comercialização uma vez que não há estudos a literatura que faça essa análise para o mercado interno de etanol. Os resultaram demonstraram que um aumento de $20 \%$ no custo das tarifas significa uma perda de 3,9\% do mercado que temos hoje, enquanto uma queda da ordem de $20 \%$ nas tarifas representa um aumento de $4,8 \%$ no fluxo comercializado, ou seja, um aumento nas receitas da ordem de US\$ 303 milhões.

Atualmente já existe uma discussão sobre uniformizar a cobrança da alíquota de ICMS, se as alíquotas para os todos os estados fossem reajustadas para o valor mínimo (7\%) como é para Minas Gerais e São Paulo, já teríamos um ganho razoável no fluxo de movimentação.

O cenário 3 é o cenário principal por juntar os custos de transporte com os valores das tarifas, formando o que chamamos de custo das transações comerciais. Esse cenário é o que apresenta o melhor resultado, uma vez que quando aumentamos o custo das transações comerciais em $20 \%$ há uma perda no fluxo movimentado da ordem de $4,3 \%$, uma perda de mais de R $\$ 257$ milhões em comparação com as receitas obtidas com venda de etanol no ano de 2016.

Ainda no cenário 3 , se diminuirmos $20 \%$ o custo das transações comerciais, o fluxo comercializado aumenta em 5,3\%, representando um ganho de US\$ 443 milhões em receitas provenientes do mercado do etanol.

Se comparados os três cenários alternativos, percebe-se que reduções nas tarifas causam maior efeito no mercado de etanol do que reduções no custo de transporte, isso nos mostra o quanto a política de taxação precisa ser revista, uma vez que ganhos de competitividade podem ser garantidos com novas alíquotas e taxas de importação.

Embora o choque no custo de transporte não tenha sido o grande destaque desse modelo não podemos deixar de fora de durante o período de pesquisa para esse artigo tivemos uma greve dos caminhoneiros que parou o país e principalmente o abastecimento de etanol nos postos por mais de uma semana, isso mostra o quão frágil é o sistema de transporte nacional e o nosso nível de dependência do modal rodoviário.

A grande contribuição deste trabalho para o mercado de etanol em geral é a aplicação do modelo matemático da forma como foi implementado, já que modelos que levam em conta os custos das transações comerciais nunca foram desenvolvidos para o produto etanol.

Além disso, os resultados apresentados nas simulações podem auxiliar em formulação de políticas que beneficiem vários segmentos do setor, a iniciativa privada que pode apurar melhor resultado com redução de custos, o governo que pode se beneficiar com uma possível reforma tributária, produtores rurais podem ter a matéria-prima mais valorizada e a tonelada pode enfim ter um preço mais justo e as usinas podem conseguir o capital necessário para se desafogarem das dívidas e recuperações judiciais.

Além disso, mesmo que ocorra uma reforma brasileira na tributação, se faz necessário o desenvolvimento de novos parceiros comerciais internacionais, uma vez que as exportações de etanol estão menores a cada ano. O Japão é um exemplo disso, até 2016 o Japão não aparecia como potencial comprador do etanol brasileiro e hoje já ocupa a posição de quarto maior comprador de etanol brasileiro. 


\section{REFERÊNCIAS BIBLIOGRÁFICAS}

[1] ALVIM, A. M. Os impactos dos novos acordos de livre comércio sobre o mercado de arroz no Brasil: um modelo de alocação espacial e temporal. 2003, 221 p. Tese (Doutorado em Economia) -Universidade Federal do Rio Grande do Sul, Porto Alegre.

[2] ANP - Agência Nacional do Petróleo. Abastecimento em números. Ano 13, n58. 2018 Disponível em:< http://www.anp.gov.br/images/publicacoes/boletins-anp/Boletim_Abastecimento/58/ Boletim_n58.pdf $>$ Acesso em jan de 2019

[3] ANTT - Agência Nacional de Transportes Terrestres. Relatório de Gestão 2016. Brasília, 2016, 333p.

[4] BEIRAL, P. R. S. (2011). O mercado brasileiro de etanol: concentração e poder de mercado sob a ótica da nova organização industrial empírica. São Paulo, 2011. 108f. Dissertação (Mestrado em Economia Aplicada) - Escola Superior de Agricultura Luiz de Queiroz, Universidade de São Paulo, Piracicaba, SP, Brasil.

[5] BOFF, H. P., O mercado interno do etanol: Modelo e Estimação do Preço de Equilíbrio, Seminários de pesquisa, 17032, IE/UFRJ, Rio de Janeiro, 17 Março. 2009.

[6] BRASIL. AGROSTAT: Estatísticas de Comércio Exterior do Agronegócio Brasileiro. Disponível em: http://sistemasweb.agricultura.gov.br/pages/AGROSTAT.html. Acesso em 10 ago. 2017.

[7] CAETANI, M. I. da R. Os efeitos dos custos das transações comerciais e do livre comércio no mercado internacional da soja. 2014. 78 f. Dissertação (Mestrado) - Curso de Economia, Pontifícia Universidade Católica do Rio Grande do Sul, Porto Alegre, 2014.

[8] CEPEA. Centro de Estudos Avançados em Economia Aplicada. Indicador Semanal do Etanol Hidratado Combustível Cepea/Esalq - São Paulo. 2017. Disponível em https://www.cepea.esalq.usp.br/br/indicador/etanol.aspx Acessado em 2 de junho de 2017.

[9] COLETI, J. de C. (2015) Transporte e intermodalidade do etanol brasileiro : uma aplicação de um modelo de equilíbrio parcial. 2015. 117 p. Dissertação (Mestrado) Desenvolvimento Econômico, Universidade Estadual de Campinas, Campinas, SP, Brasil.

[10] ENKE, S. Equilibrium among Spatially Separated Markets: Solution by Electrical Analogue. Econometrica, v. 19, 1951, p. 40-47.

[11] ESTADOS UNIDOS. Departament of Agriculture. USDA agricultural projeections to 2022. Washington, $2017 . \quad$ DC, Disponível http://www.ers.usda.gov/publications/oce-usda-agricultural-projections/ oce131.aspx\#.U_eYrfldVqU Acesso em 04 ago. 2017.

[12] INFORME SIFRECA. Sistema de informações de fretes para cargas agrícolas. Piracicaba: [s.n.], 2017.

[13] ITA. International Trade Adminsitrations. 2016 Top Markets Report Renewable Fuels -Sector Snapshot 2016. Disponível em: https://business.gov.nl/regulation/excise-dutyconsumption-tax/. Acessado em 2 jun 2017. 
[14] KRUGMAN , P., WELLS, R. Economics. $3^{\text {a }}$ Edição, Editora MacMillan, New York, 2012.

[15] LUCHANSKY, M. S. e MONKS, J. Supply and Demand Elasticities for the United States Ethanol Market, Energy Economics ,v. 31, n. 3, p. 403-410, 2009.

[16] MARTINS, R. S.; CAIXETA FILHO, J. V. Subsídios à tomada de decisão da escolha da modalidade para o planejamento dos transportes no estado do Paraná. Revista de Administração Contemporânea. Curitiba, v.3, n.2, p.75-96, mai./ago. 1999.

[17] MILANEZ, A. Y.; NYKO, D.; GARCIA, J. L. F.; XAVIER, C. E. O.. Logística para o etanol: situação atual e desafios futuros. BNDES Setorial, Rio de Janeiro: BNDES, n. 31, jun. 2010.

[18] NAGURNEY, A., DONG, J., ZHANG, D. A supply chain network equilibrium model. Transportation Research Part E. No 38. P. 281-303, 2002.

[19] NETHERLANDS. Excise duty and consumption tax 2016. Disponível em: https://business.gov.nl/regulation/excise-duty-consumption-tax/ Acessado em jun 2017.

[20] OECD. Organisation for Economic Co-operation and Development. Maritime Transport Costs. Disponível em: < https://stats.oecd.org/index.aspx? DatasetCode=MTC $>$ Acessado em dez 2017.

[21] OLIVEIRA, A. L. R. O., ALVIM, A. M. The supply chain of Brazilian maize and soybeans: the effects of segregation on logistics and competitiveness. The International Food and Agribusiness Management Review, 20 (1): 45-61, 2017.

[22] OLIVEIRA, A., SILVEIRA, J., \& ALVIM, A Cartagena Protocol, Biosafety and Grain Segregation: The Effects on the Soybean Logistics in Brazil. Journal of Agricultural Research and Development, 2(1), 17-30, 2012.

[23] OLIVEIRA, A.M.K. e FILHO, J.V.C. (2007) 'Potencial da logística ferroviária para exportação de açúcar em São Paulo: recomendações de localização para armazéns intermodais', Revista de Economia e Sociologia Rural, Vol. 45, No. 4, pp.823-853.

[24] ORTÚZAR, J. D.; WILLUMSEN, L. G. Modelling transport. 2nd. ed. Chichester: John Wiley \& Sons, 1995.

[25] PARIS, Q., DROGUÉ, S., ANANIA,G. Calibrating spatial models of trade. Econ. Model., 28 (6) pp. 2509-2516, (2011).

[26] RENEWABLE FUELS ASSOCIATION (RFA). Monthly U.S. Fuel Ethanol Production/Demand. Disponível em: <http://ethanolrfa.org/pages/monthly-fuelethanol-production-demand>. Acesso em 5 ago de 2018.

[27] SAMUELSON, P.A. Spatial Price Equilibrium and Linear Program. American Economic Review, v.42, 1952, p.283-303.

[28] SILVEIRA, F. G. O Trabalho Agrícola no Boom do Agronegócio e na Expansão das Políticas para a Pequena Agricultura. Nota Técnica, Mercado de Trabalho - IPEA, $\mathrm{n}^{\mathrm{0}}$ 63, out de 2017, p.27 -38.

[29] TAKAYAMA, T. AND JUDGE, G.G. Spatial Equilibrium and Quadratic Programming. Journal of Farm Economics, 46, 67-93. 1964.

[30] TAKAYAMA, T.; JUDGE, G.G. Spatial and Temporal Price and Allocation Models. Amsterdan: North Holland Publishing Co., 1971. 
[31] UNIÃO DA INDÚSTRIA DE CANA-DE-AÇÚCAR (UNICA). Histórico de produção e moagem. Disponível em: <http://www.unicadata.com.br/historico-de-producao-emoagem.php?idMn=31\&tipoHistorico=2> . Acesso em 3 jun de 2017.

[32] WAQUIL, P. D. O setor agrícola na Área de Livre Comércio das Américas: desafios da integração regional. Análise Econômica, Porto Alegre : UFRGS/FCE, n. 34, p. 5774, 2000.

[33] WEDEKIN, I. Gerenciamento do risco no agronegócio. In: BUANAIN, A. M.; VIEIRA, P. A.; CURY, W. J. M. (Org.). Gestão do risco e seguro na agricultura brasileira. Rio de Janeiro: Funenseg, 2011. p. 47-55.

As Referências Bibliográficas devem seguir as normas da ABNT/NBR 6023. 